\title{
Importância da hemoglobina glicada no controle do diabetes mellitus e na avaliação de risco das complicações crônicas
}

\author{
Glycohemoglobin importance in the diabetes mellitus control and in the risk evaluation of chronic complications
}

Nairo Massakazu Sumita' ; Adagmar Andriolo²

\section{unitermos \\ Diabetes mellitus}

Hemoglobina glicada

A1C

Controle glicêmico

\section{resumo}

0 diabetes mellitus (DM) continua sendo objeto de pesquisa, dadas as constantes informaccoes que os estudos clínicos e os novos recursos laboratoriais incorporam à prática médica a cada dia e com maiores rapidez e eficiência. Níveis glicêmicos persistentemente elevados são danosos ao organismo e o descontrole prolongado resulta em complicações, incluindo danos em diversos tecidos, perda da função normal e falência de vários órgãos. Para 0 acompanhamento do portador de DM, a hemoglobina glicada (A1C) tem se firmado como ferramenta útil depois de ter sido validada pelos dois estudos clínicos mais importantes sobre a avaliacão do impacto do rígido controle glicêmico sobre a incidência e a progressão das complicacôes do diabetes: 0 Diabetes Control and Complications Trial (DCCT, 1993) e o United Kingdom Prospective Diabetes Study (UKPDS, 1998). Essas pesquisas demonstraram que manter o nível de $\mathrm{A} 1 \mathrm{C}$ abaixo de $7 \%$ reduz o risco de desenvolvimento das complicações dessa doença. 0 Grupo Interdisciplinar de Padronização da Hemoglobina Clicada - A1C, criado pela associacão de diversas sociedades cientificas e farmacêuticas do Brasil, publicou, em 2004, um documento de posicionamento oficial acerca da importância da Ả1C para a avaliação do controle glicêmico, abordando os principais aspectos clínicos e laboratoriais, incluindo as condições de variação pré-analítica e analítica. Foram estabelecidas as recomendaçốes a respeito das indicaçốes do teste e dos valores ideais de controle para adultos, crianças e idosos. Segundo este posicionamento, os testes de A1C devem ser realizados pelo menos duas vezes ao ano por todos os portadores de DM. Quando os resultados não forem adequados e/ou forem realizadas alterações no esquema terapêutico, a dosagem deve ser feita depois de três meses. A dosagem está indicada tanto para os portadores de diabetes mellitus tipo 1 (DM1) quanto tipo 2 (DM2), sendo que a meta a ser atingida, representando efetivo controle, em ambas as condiçôes é abaixo de $7 \%$, tanto no adulto como no adulto jovem. Para as crianças durante a fase pré-puberal, o nível aceitável de A1C é de até $8 \%$ e, na fase puberal, até $8,5 \%$. Nos pacientes idosos, a A1C de até $8 \%$ é considerada apropriada, uma vez que a tentativa de um controle muito rígido da glicemia nesta faixa etária, assim como nas fases pré-puberal e puberal, pode induzir a efeitos colaterais indesejados, como, por exemplo, hipoglicemia. Para a paciente gestante não está indicado o acompanhamento do controle glicêmico pela dosagem de A1C, sendo mais eficiente o controle dos níveis das glicemias de jejum e duas horas após as refeiçôes e a dosagem de frutosamina, que corresponde ao coniunto das proteínas plasmáticas glicosadas. O grande diferencial da A1C em relacão a glicemia de jejum é que os níveis daquela variam mais lentamente, dependendo da meia-vida das hemácias, portanto não retornam ao normal imediatamente depois da normalização da glicose no sangue. 0 tempo para que a A1C atinja os níveis adequados após um período de hiperglicemia é de aproximadamente dez semanas. Assim, a repetição do exame de AIC para avaliar a eficácia de um tratamento deve ser realizada somente dois a três meses depois do início ou da modificacão do esquema terapêutico. Doenças que alteram a sobrevida das hemácias, como anemia hemolítica e hemorragia, por reduzirem sua vida média, podem resultar em valores falsamente baixos de $\mathrm{Hb} A 1 C$, enquanto as anemias por carência de ferro, de vitamina B12 ou de folato, que aumentam a vida média das hemácias, resultam em valores falsamente elevados. Na dependência da metodologia utilizada, outras condições clínicas podem interferir no resultado de A1C, como hipertrigliceridemia, hiperbilirrubinemia, uremia, alcoolismo crônico, uso prolongado de opiáceos ou de salicilatos. O posicionamento oficial brasileiro recomenda a utilização de métodos rastreáveis do Diabetes Control and Complications Trial (DCCT), conforme certificado pelo National Clycohemoglobin Standardization Program (NCSP), e estimula a participação em programas de ensaios de proficiência espeć́ficos para AIC.

\section{abstract}

Diabetes mellitus remains a subject of study due to the fact that clinical trials and new laboratory resources have increasingly added updated information to medical practice. High glycemic levels are harmful and their persistence results in complications such as tissue damage, loss of normal function and failure of several organs. Glycated hemoglobin control has been a useful tool to monitor diabetic patients, and this analyte was validated by two major clinical studies about the impact assessment of rigid glycemic control on the incidence and progression of diabetes complications: Diabetes Control and Complications Trial (DCCT, 1993) and United Kingdom Prospective Diabetes Study (UKPDS, 1998). These studies showed that glycated hemoglobin level below 7\% reduces the risk of complications in diabetes. In 2004, the Interdisciplinary Group of Standardization of Clycated Haemoglobin-A1C, a group of specialists from scientific societies and pharmaceutical companies in Brazil, published an official statement about the importance of glycated hemoglobin for the assessment of glycemic control. It discusses the clinical and laboratory aspects, which includes pre-analytical and analytical variations. The recommendations for the use of the test and the ideal control levels for adults, children and elderly people were established. According to this document, A1C tests should be performed at least twice a year by all patients with diabetes mellitus. However, when the results are not appropriate and/or changes are made in the therapeutic scheme, the test should be performed after three months. It is recommended for individuals with diabetes types 1 and 2 and the goal to be achieved is below $7 \%$ for effective control in both adults and youngsters. For children during the prepubertal stage the acceptable level of A1C is up to $8 \%$ and in pubertal stage, up to 8.5\%. In elderly patients, A1C up to $8 \%$ is considered appropriate insofar as the attempt to a rigid glycemic level in this age group as well as in prepubertal and pubertal stages may cause side effects such as hypoglycemia. Glycated hemoglobin control is not recommended for pregnant women. The fasting blood glucose test, the glucose level after meals and the fructosamine determination, which corresponds to the group of glycated serum proteins, are more efficient in this case. The main difference between A1C and fasting blood glucose is that the levels of A1C vary more slowly, depending on the half-life of red blood cells. Therefore, they do not return to normality immediately after the normalization of glucose in the blood. The time to reach appropriate levels of $\mathrm{A1C}$ after a period of hyperglycemia is approximately 10 weeks. Consequently, A1C exams should be repeated only two to three months after the beginning or the modification of the therapeutic scheme in order to assess its effectiveness. Diseases that affect the survival of red blood cells, such as hemolytic anemia and hemorrhage, may result in false low A1C values due to the reductions of their half-life. On the other hand, anemia caused by iron, vitamin B12 or folate deficiencies, which increase the half-life of red blood cells, may result in false elevated A1C values. Depending on the methodology applied, other medical conditions, such as hypertriglyceridemia, hyperbilirubinemia, uremia, chronic alcoholism and chronic use of opiates or salicylates, may interfere in the results of A1C. Not only is the Brazilian official recommendation the use of DCCT traceable methods, certified by the National Clycohemoglobin Standardization Program (NCSP), but it also encourages participation in specific proficiency testing programs of glycated hemoglobin. key words

Diabetes mellitus

Glycated hemoglobin

A1C

Glycemic control

1. Doutor e professor assistente da disciplina de Patologia Clínica da Faculdade de Medicina da Universidade de São Paulo (FMUSP); diretor do Serviço de Bioquímica Clínica da Divisão de Laboratório Central do Hospital das Clínicas da FMUSP; assessor médico na área de bioquímica clínica do Fleury Medicina e Saúde.

2. Professor livre-docente do Departamento de Medicina da Escola Paulista de Medicina da Universidade Federal de São Paulo (EPM/UNIFESP); consultor médico do Fleury Medicina e Saúde. 


\section{Introdução}

O termo hemoglobina glicada define um grupo de substâncias formadas a partir da reação entre a hemoglobina $\mathrm{A}$ $(\mathrm{HbA})$ e um açúcar. O componente mais importante deste conjunto é a fração $\mathrm{A} 1 \mathrm{C}$, na qual há um resíduo de glicose ligado ao grupo amino terminal (resíduo de valina) de uma ou de ambas as cadeias beta da HbA. A ligação entre a HbAe a glicose é o produto de uma reação não-enzimática definida como glicação. Por esta razão, obedecendo à nomenclatura química, o termo correto é hemoglobina glicada, devendo ser abandonado o termo hemoglobina glicosilada. A primeira fase da reação entre a glicose e a hemoglobina é reversível e origina um composto intermediário denominado pré-A1C ou base de Schiff. A segunda fase é irreversível e resulta em um composto estável denominado A1C. A membrana da hemácia é altamente permeável à molécula de glicose, fazendo com que a hemoglobina presente no seu interior fique exposta praticamente à mesma concentração da glicose plasmática. A glicação ocorrerá em maior ou menor grau, conforme o nível de glicemia. A hemoglobina glicada permanece dentro das hemácias e a sua concentração, num determinado momento, dependerá, basicamente, da taxa glicêmica média e da meia-vida das hemácias ${ }^{(4,8,9,11,16)}$.

Como ocorre com a maioria dos parâmetros bioquímicos, o intervalo de referência para a A1C depende da metodologia utilizada. Considerando-se o método de cromatografia líquida de alto desempenho (CLAD) ou high performance liquid chromatography (HPLC), na língua inglesa ${ }^{(6)}$, o intervalo de referência da $\mathrm{A} 1 \mathrm{C}$ nos indivíduos não-diabéticos é de $4 \%$ a $6 \%$. Níveis elevados de $\mathrm{A} 1 \mathrm{C}$ não fazem, obrigatoriamente, diagnóstico de diabetes mellitus (DM), mas permitem a estimativa da glicemia média pregressa, medida esta que possibilita uma avaliação da qualidade do controle glicêmico.

\section{A importância da hemoglobina glicada no controle diabetes mellitus}

No DM, a hiperglicemia persistentemente prolongada é bastante nociva ao organismo. Existe estreita relação entre níveis elevados de glicose no sangue e surgimento das complicações do diabetes. O descontrole permanente acarreta, no decorrer dos anos, uma série de complicações orgânicas, resultando em danos teciduais, perda de função e falência de vários órgãos ${ }^{(13,14)}$.

A dosagem da A1C tem grande importância na avaliação do nível de controle do DM, sendo indicada para todos os portadores de diabetes ${ }^{(13,14)}$. Importante frisar que ainda não existem evidências que justifiquem a realização desse exame com finalidade diagnóstica, mas apenas para acompanhar o tratamento ${ }^{(1)}$.

A dosagem da A1C passou a ser considerada parâmetro essencial na avaliação do controle do DM após a publicação dos resultados de dois grandes estudos clínicos: Diabetes Control and Complications Trial (DCCT), em 1993(6), e United Kingdom Prospective Diabetes Study (UKPDS), em 1998(19), relativos ao diabetes mellitus tipos 1 e 2 (DM1 e DM2), respectivamente. Esses estudos demonstraram, claramente, que manter o nível de A1C abaixo de $7 \%$ no portador de diabetes reduz significativamente o risco de desenvolvimento das complicações micro e macrovasculares da doença em relação ao paciente cronicamente descontrolado ${ }^{(6,19)}$.

Como a quantidade de glicose ligada à hemoglobina é diretamente proporcional à concentração média de glicose no sangue, e como os eritrócitos têm meia vida de aproximadamente 120 dias, a medida da quantidade de glicose ligada à hemoglobina pode fornecer uma avaliação do controle glicêmico médio no período de 60 a 90 dias que antecedem a coleta de sangue para o exame $\mathrm{e}^{(7-9,11)}$.

Ao interpretar o resultado da dosagem da A1C, é necessário considerar que os níveis médios mais recentes da glicemia são os que mais influenciam no valor da A1C. Aproximadamente $50 \%$ da A1C são formados no mês precedente ao exame, $25 \%$ no mês anterior a esse e os $25 \%$ remanescentes, no terceiro ou quarto mês que precede a coleta da amostra ${ }^{(17)}$.

Segundo os estudos do DCCT, existe estreita correlação entre os níveis de $\mathrm{A} 1 \mathrm{C}$ e os valores médios de glicose plasmática, conforme descrito na Tabela ${ }^{(6)}$. Elevação de $1 \%$ na hemoglobina glicada corresponde a, aproximadamente, um aumento médio de 25 a $35 \mathrm{mg} / \mathrm{dl}$ na glicemia. Uma elevação de $3 \%$ indica que a glicemia média mantém-se acima de $200 \mathrm{mg} / \mathrm{dl}^{(3,6)}$.

As dosagens de glicose e de A1C são complementares para a avaliação do controle do DM, pois fornecem informações distintas acerca dos níveis de glicose sangüínea. Os resultados de $\mathrm{A} 1 \mathrm{C}$ refletem a glicemia média no intervalo de dois a três meses precedentes à coleta, enquanto a dosagem de glicose reflete a glicemia unicamente no momento da coleta da amostra de sangue ${ }^{(13,14)}$.

No Brasil, o Grupo Interdisciplinar de Padronização da Hemoglobina Glicada - A1C publicou, em 2003, um documento de posicionamento oficial sobre a importância da 


\begin{tabular}{ccc} 
Tabela & \multicolumn{2}{c}{$\begin{array}{c}\text { Correlação entre os níveis de A1C e os } \\
\text { níveis médios de glicose plasmática }\end{array}$} \\
\hline Nível de & Interpretação & $\begin{array}{c}\text { Glicose média } \\
(\mathrm{mg} / \mathrm{dl})\end{array}$ \\
A1C (\%) & & 65 \\
4 & & 100 \\
5 & Faixa dos valores de & 135 \\
6 & referenciais & 170 \\
7 & Meta a ser alcançada no & \\
& paciente diabético & 205 \\
8 & & 240 \\
9 & & 275 \\
10 & Necessidade de & 310 \\
12 & intervenção terapêutica & 345 \\
\hline
\end{tabular}

hemoglobina glicada para a avaliação do controle glicêmico em pacientes com DM. Esse documento foi revisado em 2004, abordando os aspectos clínicos e laboratoriais relacionados com esse importante parâmetro laboratoria|l ${ }^{(8,9)}$ e estabelecendo uma série de recomendações acerca da utilidade do teste, bem como valores ideais de controle para adultos, crianças e idosos.

\section{Padronização dos métodos para dosagem da A1C}

Existem numerosas metodologias para dosagem da A1C, resultando em ampla variabilidade nos valores referenciais. No entanto, o valor de 7\% como nível adequado para controle do diabetes foi validado pelos estudos do DCCT com base no método de CLAD. Com a finalidade de minimizar os problemas de interpretação dos resultados da $\mathrm{A} 1 \mathrm{C}$ pelas diferentes metodologias foi criada nos EUA uma entidade denominada National Glycohemoglobin Standardization Program (NGSP) (http://www.missouri.edu/diabetes/ngsp. $\mathrm{html})^{(10)}$. Esse programa avalia os métodos disponíveis no mercado mundial com o intuito de verificar se produzem resultados compatíveis com a metodologia utilizada pelo DCCT. Caso essa equivalência não seja observada, avalia-se a possibilidade de ser estabelecido um cálculo matemático para correção do resultado, tornando o método rastreável em relação ao utilizado no DCCT. O posicionamento oficial recomenda que os laboratórios clínicos utilizem, preferencialmente, os métodos de ensaio certificados pelo NGSP, com rastreabilidade de desempenho analítico do método utilizado no $D C C T^{(8,9)}$. Além disso, é preconizado que os laboratórios que realizam essa rotina participem de programas de ensaios de proficiência específicos para a A1C implementados por entidades oficiais, como, por exemplo, a Sociedade Brasileira de Patologia Clínica/Medicina Laboratorial $(\mathrm{SBPC} / \mathrm{ML})^{(8,9)}$

\section{Metas a serem alcançadas nos níveis de $\mathrm{A} 1 \mathrm{C}$ e freqüência para realização do exame}

Segundo o documento de posicionamento oficial, os testes de A1C necessitam ser realizados pelo menos duas vezes ao ano por todos os portadores de diabetes. Quando eles não conseguem atingir um controle adequado, a recomendação é realizar a dosagem da $\mathrm{A} 1 \mathrm{C}$ a cada três meses ${ }^{(8,9)}$. A dosagem de A1C está indicada tanto para os diabéticos do tipo 1 como para os do tipo 2 , sendo que a meta a ser atingida para o efetivo controle do DM, em ambas as condições, é abaixo de $7 \%$ tanto no adulto como no adulto jovem ${ }^{(8,9)}$.

Os valores adequados de A1C para crianças e adolescentes diabéticos ainda não estão perfeitamente estabelecidos, no entanto é inegável a necessidade de se estabelecerem níveis de referência e padrões de bom controle para essas faixas etárias. A estratégia a ser adotada para controle adequado nos níveis de glicose deve garantir crescimento e desenvolvimento adequados; baixo risco de hipoglicemia, principalmente em crianças com menos de 8 anos de idade, quando o desenvolvimento neurológico ainda não está completo; e minimizar o risco de desenvolvimento precoce das complicações crônicas ${ }^{(9)}$. Em crianças, na fase pré-puberal, o nível aceitável de $\mathrm{A} 1 \mathrm{C}$ é de até $8 \%$, e na fase puberal, inferior a $8,5 \%$. A meta para $\mathrm{A} 1 \mathrm{C}$ inferior a $7 \%$ deverá ser adotada a partir da fase final da puberdade ${ }^{(9)}$. A freqüência de realização das dosagens de $\mathrm{A} 1 \mathrm{C}$ na infância e na adolescência depende das disponibilidades locais e do nível de controle alcançado, podendo variar de duas a quatro vezes ao ano $^{(9)}$.

Nos idosos, o nível de A1C de até $8 \%$ é considerado apropriado, uma vez que a tentativa de controle mais rígido da glicemia nesta faixa etária pode induzir a efeitos colaterais indesejados, como hipoglicemia severa ${ }^{(9)}$.

Na gravidez, a dosagem de $\mathrm{A} 1 \mathrm{C}$ não está indicada para monitoração. Nessa situação, é muito mais eficiente um controle rigoroso dos níveis das glicemias de jejum e de duas horas após as refeições. $\mathrm{O}$ acompanhamento pré-natal da paciente com DM é fundamental para minimizar os riscos de complicações, principalmente o aborto espontâneo ${ }^{(9)}$. 
Os níveis de $\mathrm{A} 1 \mathrm{C}$ não retornam ao normal imediatamente após a normalização da glicose no sangue. O intervalo para que a A1C atinja os níveis estáveis após um episódio de descontrole é de aproximadamente dez semanas. Assim, a repetição do exame de A1C para avaliar a eficácia de um tratamento deve ser realizada somente dois a três meses depois do início ou da modificação do esquema terapêutico. Antes desse tempo, os níveis de A1C praticamente não sofrerão grandes mudanças( ${ }^{(9)}$. A avaliação dos níveis médios de glicemia em períodos mais curtos pode ser obtida pela dosagem de frutosamina, a qual é capaz de informar acerca do controle glicêmico das últimas quatro a seis semanas, sendo útil na avaliação da eficácia de uma mudança terapêutica(4).

\section{Aspectos laboratoriais}

Em relação aos aspectos analíticos, algumas situações clínicas podem gerar interferências na análise da hemoglobina glicada. A idade, o sexo, a origem étnica ou a falta de jejum não afetam significativamente os resultados da $\mathrm{A} 1 \mathrm{C}^{(7)}$.

As variantes genéticas da hemoglobina, como, por exemplo, as hemoglobinas $S$ ou $C$, na condição heterozigótica, podem produzir interferências na medida da hemoglobina glicada, resultando em valores falsamente elevados ou diminuídos, de acordo com o tipo de método de ensaio utilizado. A dosagem de A1C não se aplica nas condições de homozigose para hemoglobinas anômalas, por qualquer metodologia, pois nesses casos a hemoglobina A está ausente. Nessas circunstâncias, o exame alternativo, como a frutosamina, pode ser útil $(4,7-9,11)$.

Outras variantes genéticas de hemoglobina também podem interferir na dosagem de hemoglobina glicada, principalmente nas metodologias que não estão aptas a identificá-las adequadamente. São conhecidos mais de 700 tipos de variantes de hemoglobina. O Grupo Interdisciplinar de Padronização da Hemoglobina Glicada - A1C recomenda que os resultados abaixo do limite inferior do intervalo de referência ou acima de $15 \%$ sejam sempre confirmados por outra metodologia, visando, principalmente, a identificação de eventuais interferentes dessa natureza( ${ }^{(9)}$. O estudo do perfil das hemoglobinas para rastreamento das hemoglobinopatias também está indicado nessas situações ${ }^{(9)}$.

As doenças que alteram o tempo de sobrevida das hemácias, como anemia hemolítica e hemorragia, que reduzem a sobrevida das hemácias, podem resultar em valores falsamente baixos ${ }^{(5,7-9)}$. A presença de grandes quantidades de vitaminas $\mathrm{C}$ e $\mathrm{E}$ é descrita como um dos fatores que po- dem induzir a resultados falsamente diminuídos por inibir a glicação da hemoglobina ${ }^{(5,7-9)}$.

Nos estados de anemia por carência de ferro, de vitamina B12 ou de folato, em que ocorre aumento da sobrevida das hemácias, pode ser observada falsa elevação dos valores de $\mathrm{A} 1 \mathrm{C}^{(5,7-9)}$. A presença de hemoglobinas quimicamente modificadas, como, por exemplo, a hemoglobina carbamilada associada à uremia e a hemoglobina acetilada, formada após a ingestão de grandes doses de salicilatos, pode elevar falsamente os resultados ${ }^{(5,7-9)}$. Outras condições clínicas que podem elevar o resultado da A1C são hipertrigliceridemia, hiperbilirrubinemia, alcoolismo crônico e uso prolongado de opiáceos ${ }^{(5,7-9)}$.

Dependendo da metodologia utilizada, a pré-A1C ou base de Schiff, que é a fração lábil da hemoglobina glicada, pode ser um importante interferente na dosagem, ainda que as metodologias mais modernas permitam a remoção desse interferente ${ }^{(7,11)}$.

\section{Novos conceitos e tendências}

Recentemente, algumas das mais importantes instituições mundiais ligadas ao estudo do diabetes, como Associação Americana de Diabetes (ADA), Associação Européia para o Estudo do Diabetes, Federação Internacional de Química Clínica e Medicina Laboratorial (IFCC) e Federação Internacional de Diabetes (IDF), iniciaram discussões para a definição de um método de referência para dosagem da hemoglobina glicada visando uma padronização mundial do ensaio para $\mathrm{A} 1 \mathrm{C}^{(3)}$.

As seguintes propostas foram aprovadas pelo grupo: 1. a dosagem de $\mathrm{A} 1 \mathrm{C}$ necessita de uma padronização mundial, incluindo a definição de um método de referência e o modo adequado para expressar os resultados;

2. o novo método proposto pelo IFCC serviria como âncora para a padronização da medida da A1C. Os métodos atualmente disponíveis para uso no laboratório clínico continuariam válidos, porém necessitariam ser referendados pelo método do IFCC;

3. a hemoglobina glicada seria expressa em duas unidades: $\mathrm{mmol} / \mathrm{mol}$ (IFCC) e percentual (NGSP);

4. a hemoglobina glicada seria transformada num valor correspondente à glicose média por meio de uma equação matemática. Esse resultado de glicose média derivada da A1C seria incorporado ao laudo do exame, visando facilitar a interpretação clínica do resultado.

O laudo final de um exame de hemoglobina glicada seria composto por três resultados: $\mathrm{A} 1 \mathrm{C}$ na unidade de 
concentração definida pelo IFCC ( $\mathrm{mmol} / \mathrm{mol})$, A1C na unidade usual em conformidade com o NGSP (\%) e sob a forma de concentração de glicose média derivada da A1C $(\mathrm{mg} / \mathrm{dl})$.

\section{Conclusão}

Atualmente, a hemoglobina glicada é o exame mais importante no controle do DM tipos 1 e 2 e na avaliação de risco das complicações crônicas.

No adulto, níveis de A1C acima de 7\% estão associados a risco progressivamente maior de complicações crônicas.

A meta a ser atingida para o efetivo controle do diabetes é abaixo de $7 \%$ tanto no adulto como no adulto jovem. A critério médico e na dependência do tipo de paciente (crianças e idosos), o alvo pode ser ajustado em função do grau de risco de eventos de hipoglicemia.
A dosagem de hemoglobina glicada não está indicada para pacientes gestantes.

As dosagens devem ser realizadas pelo menos duas vezes ao ano para todos os portadores de DM e quatro vezes por ano para os pacientes que se submeterem a alterações do esquema terapêutico.

O posicionamento oficial do Grupo Interdisciplinar de Padronização da Hemoglobina Glicada - A1C recomenda que os laboratórios clínicos utilizem apenas os ensaios certificados pelo NGSP, com rastreabilidade de desempenho analítico do método utilizado no DCCT.

Os laboratórios clínicos devem participar de programas de ensaio de proficiência específicos para A1C, implementados por entidades oficiais, como, por exemplo, a Sociedade Brasileira de Patologia Clínica/Medicina Laboratorial (SBPC/ML)

\section{Referências}

1. AMERICAN DIABETES ASSOCIATION: Diagnosis and classification of diabetes mellitus. Position statement. Diabetes Care, v. 27, suppl. 1, p. S4-10, 2004.

2. AMERICAN DIABETES ASSOCIATION. Tests of glycemia in diabetes. Position statement. Diabetes Care, v. 26, p. S106-S108, 2003.

3. AMERICAN DIABETES ASSOCIATION, EUROPEAN ASSOCIATION FOR THE STUDY OF DIABETES, INTERNATIONAL FEDERATION OF CLINICAL CHEMISTRY AND LABORATORY MEDICINE AND THE INTERNATIONAL DIABETES FEDERATION. Consensus statement on the worldwide standardization of the hemoglobin A1C measurement. Diabetes Care, v. 30, p. 2399-400, 2007.

4. ANDRIOLO, A.; VIEIRA, J. G. H. Diagnóstico e acompanhamento laboratorial do diabetes mellitus. In: ANDRIOLO, A. (org.). Guias de medicina ambulatorial e hospitalar/medicina laboratorial. 1. ed. São Paulo: Manole, 2008. p. 37-42.

5. BRY, L.; CHEN, P. C.; SACKS, D. B. Effects of hemoglobin variants and chemically modified derivatives on assays for glycohemoglobin. Clin Chem, v.47, p. 153-63, 2001.

6. DCCT RESEARCH GROUP. Diabetes Control and Complications Trial (DCCT). The effect of intensive treatment of intensive treatment of diabetes on the development and progression of long-term complications in insulin-dependent diabetes mellitus. N Engl J Med, v. 329, p. 977-86, 1993.

7. GOLDSTEIN, D. E. et al. Tests of glycemia in diabetes. Diabetes Care, v. 27, p. 1761-73, 2004.

8. GRUPO INTERDISCIPLINAR DE PADRONIZAÇÃO DA
HEMOGLOBINA GLICADA - A1C. Hemoglobina glicada. Posicionamento Oficial (versão 2003). A importância da hemoglobina glicada (A1C) para a avaliação do controle glicêmico em pacientes com diabetes mellitus: aspectos clínicos e laboratoriais. Disponível em: $<$ http://www.sbpc.org.br/profissional/noticia.diverso. php?id $=8 \&$ tp $=3>$.

9. GRUPO INTERDISCIPLINAR DE PADRONIZAÇÃO DA HEMOGLOBINA GLICADA - A1C. Hemoglobina glicada. Posicionamento Oficial (versão 2004). A importância da hemoglobina glicada (A1C) para a avaliação do controle glicêmico em pacientes com diabetes mellitus: aspectos clínicos e laboratoriais. Disponível em: $<$ http://www.sbpc.org.br/profissional/noticia.diverso. php?id=5\&tp=3>.

10. LITTLE, R. R.; ROHLFING, C. L.; WIEDMEYER, H. et al. The national glycohemoglobin standardization program: a five-year progress report. Clin Chem, v. 47, p. 198592, 2001

11. SACKS, D. B. Carbohydrates. In: BURTIS, C. A.; ASHWOOD, E. R.; BRUNS, D. E. Tietz textbook of clinical chemistry and molecular diagnostics. St. Louis: Elsevier Saunders, 2006. p. 837-901

12. SAUDEK, C. D.; DERR, R. L.; KALYANI, R. R. Assessing glycemia in diabetes using self-monitoring blood glucose and hemoglobin A1C. JAMA, v. 295, p. 168897, 2006.

13. SOCIEDADE BRASILEIRA DE DIABETES. Atualização Brasileira Sobre Diabetes. Versão atualizada. 2006. Disponível em: <http://www.diabetes.org.br/educacao/ docs/atualizacaodiabetes2006.pdf>.

14. SOCIEDADE BRASILEIRA DE DIABETES. Consenso 
Brasileiro Sobre Diabetes, 2002. Diagnóstico e classificação do diabetes melito e tratamento do diabetes melito do tipo 2. 2002. Disponível em: <http:// www.diabetes.org.br/educacao/docs/Consenso_atual_ 2002.pdf>.

15. STEFFES, M. et al. DCCT/EDIC RESEARCH GROUP. Hemoglobin A1C measurements over nearly two decades: sustaining comparable values throughout the diabetes control and complications trial and the epidemiology of diabetes interventions and complications study. Clin Chem, v. 51, p. 753-58, 2005.

16. SUMITA, N. M.; ANDRIOLO, A. Importância da determinação da hemoglobina glicada no monitoramento do paciente portador de diabetes mellitus. J Bras Patol Med Lab, v. 42, editorial, 2006.

17. TAHARA, Y.; SHIMA, K. The response of GHb to stepwise plasma glucose change over time in diabetic patients. Diabetes Care, v. 16, p. 1313-4, 1993.

18. THE AMERICAN DIABETES ASSOCIATION, EUROPEAN ASSOCIATION FOR THE STUDY OF DIABETES, INTERNATIONAL FEDERATION OF CLINICAL CHEMISTRY AND LABORATORY MEDICINE AND THE INTERNATIONAL DIABETES FEDERATION. CONSENSUS COMMITTEE. Consensus statement on the worldwide standardization of the hemoglobin A1C measurement. Diabetes Care, v. 30, p. 2399-400, 2007.

19. UK PROSPECTIVE DIABETES STUDY GROUP. Intensive blood glucose control with sulphonylureas or insulin compared with conventional treatment and risk of complications in patients with type 2 diabetes. Lancet, v. 352, p. 837-53, 1998. 